\title{
Burst Adaptive Equalization of Binary Data
}

\author{
Arto Kantsila, Mikko Lehtokangas and Jukka Saarinen \\ Tampere University of Technology, Signal Processing Laboratory, \\ P.O. Box 553, FIN-33101 Tampere, Finland, Email: artok@cs.tut.fi
}

\begin{abstract}
Certain telecommunication systems, like GSM, transmit data as bursts through a communication channel. In adverse conditions, the channel can distort the original signal to such an extent that without any channel equalization, the received signal is useless. This paper considers the burst adaptive equalization of binary data. We have simulated three methods for equalization. namely, (1) linear equalizer, (2) multilayer perceptron neural network, and (3) the adaptive clustering method. The channel model used introduces both linear intersymbol interference and additive white Gaussian noise. This type of channel can distort the original signal so that nonlinear channel equalization is needed. Simulation results are presented for both fixed channel and altering channel. In the results, a neural network equalizer and an adaptive clustering method are shown to outperform a conventional linear equalizer when the bit error rate is considered.
\end{abstract}

\section{Keywords}

Neural networks, telecommunication, equalization

\section{Introduction}

In recent years, digital communication technology has developed rapidly. In fact, digital communication has almost completely set aside its analog competitors in many areas. This achievement is a result of a combination of economic factors, technological advances, and demands for completely new services /1/. 
The digital baseband transmission system can be divided into three parts: (1) transmitter, (2) transmission channel, and (3) receiver. The transmitter forms the signal to be transmitted and transmits it through the channel to the receiver. The task of the receiver is to find the original information being sent. Usually the channel distorts the transmitted signal by causing, for example, intersymbol interference (ISI) and additive noise /1/. The distortions can be so severe that the receiver must equalize the received signal to compensate for the channel distortions and to be able to find the original information. Because the channel response is usually not known a priori, equalization must be done adaptively.

In this work, we have studied adaptive equalization of binary data. To be more precise, the transmitted signal considered is a burst of bits that has certain features that are similar to a GSM burst $/ 2,3 /$. The burst consists of two parts: training sequence and data sequence. The training sequence is used to adapt the equalizer, which is then used to equalize the data sequence. Here we have studied three different equalization techniques: (1) a linear equalizer, (2) a multilayer perceptron network, and (3) an adaptive clustering method. Their performance is compared to the optimal Bayesian equalizer. We have simulated channels in which the distortions are so severe that some nonlinearity must be added to the equalization process to perform equalization well. In such channels, neural networks have been found useful, see for example /4-9/. As equalization can also be seen as a classification problem $15 /$, we have also simulated adaptive clustering to perform burst adaptive equalization.

This paper is organized as follows. In Sec. 2, adaptive equalization is discussed. Three equalization methods are described in Sec. 3. Simulation results with a conventional linear equalizer. multilayer perceptron network, and adaptive clustering method are presented in Sec. 4, and they are compared with the results obtained with the optimal Bayesian equalizer. Final conclusions are given in Sec. 5 .

\section{Adaptive Equalization}

As stated earlier, the channel distorts the transmitted communication signal, for example, by causing both intersymbol interference (ISI) $/ 10 /$ and 
additive noise. ISI is usually result of the restricted bandwidth that is allocated to the channel and/or the presence of multipath distortion in the medium through which the information is transmitted /9,11/. Noise can come from the thermal noise within the receiver itself or from man-made or natural interference, such as lightning $/ 12 /$. The receiver tries to find the original data that was sent from the distorted signal by minimizing the probability of error. The design of a good receiver is even more important than that of a transmitter because we must achieve a bit error rate (BER) that is as small as possible.

\subsection{Equalization problem}

Figure 1 shows the structure of a baseband digital receiver $/ 1 /$ The task of the equalizer component in Fig. 1 is to compensate for the distortion of the channel and to decrease the effect of additive noise $/ 1,11 /$. The timing recovery component in Fig. 1 produces a synchronization signal, which is used to convert the continuous-time signal into a discrete-time signal / $1 /$. In this work, we assume that this task has been taken care of. The decision device detects the transmitted symbol sequence. Finally, the decoder forms a bit sequence from the symbol sequence.

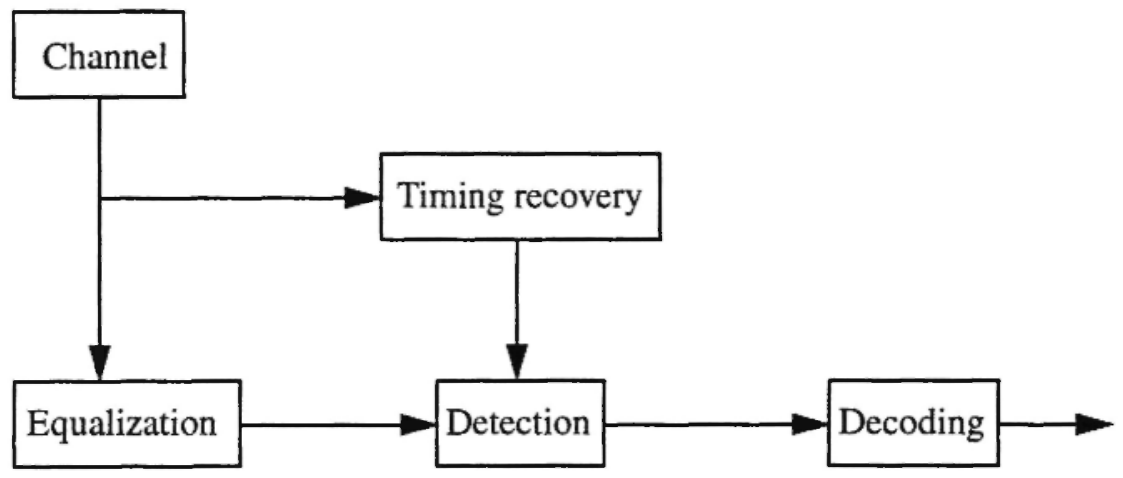

Fig. 1: Block diagram of a baseband digital receiver. 
In an often-used channel model ISI, part of the channel is modeled as a finite impulse response (FIR) filter $/ 11,13 /$. Additionally, noise is added to the signal with ISI. Thus the channel model can be described as follows $/ 1,11 /$

$$
y(t)=\sum_{i=0}^{N} h(i) a(t-i)+\varepsilon(t)
$$

where $y$ is the channel output, $a$ is the transmitted signal, $h(i)$ are the channel coefficients, $\varepsilon$ represents additive noise, and $t$ represents time. We shall consider this channel model throughout this work. Usually, the channel characteristics are not known a priori, and the channel response can be time-variant $/ 1,11 /$. Therefore, the equalizer must be adjustable to the channel response, and for time-variant channels, must be adaptive to the time variations in the channel response. Such equalizers are called adaptive equalizers.

One practical method of compensating for the ISI is to construct an approximation to the inverse of the channel with linear adaptive filters $/ 9 /$. Many linear adaptive filter algorithms are available for the training of this kind of an equalizer $/ 14 \%$. This technique. however, has a well-known disadvantage in a form of noise enhancement $/ 15 /$ because the adaptive filter does not exploit the fact that the transmitted sequence comes from a finite alphabet.

Equalization can also be seen as a classification problem, in which an equalizer is constructed as a decision-making device to reconstruct the transmitted symbol sequence as accurately as possible /5/. Figure 2 shows an example of how equalization can be viewed as a classification problem. Figure 2 presents a channel output. where the channel has been modeled as a FIR filter with additive white Gaussian noise (AWGN), as described in Eq. (1). The channel impulse response is now $\underline{h}=(h(0), h(1), \ldots, h(4))=(0.5-0.3$ $0.6-0.7-0.8$ ). AWGN has zero mean and variance $\sigma^{2}=0.001$. The transmitted data signal $a$ was given binary values +1 or -1 . The horizontal axis in Fig. 2 represents the channel output $v$ at time $t$, and the vertical axis represents the previous channel output. If the true data bit $a(t)$ corresponding to $y(t)$ is one, the point is marked as ' $x$ '. Otherwise the point is marked with ' $o$ ', meaning that the true bit $a(t)$ was minus one. As can be seen, the 


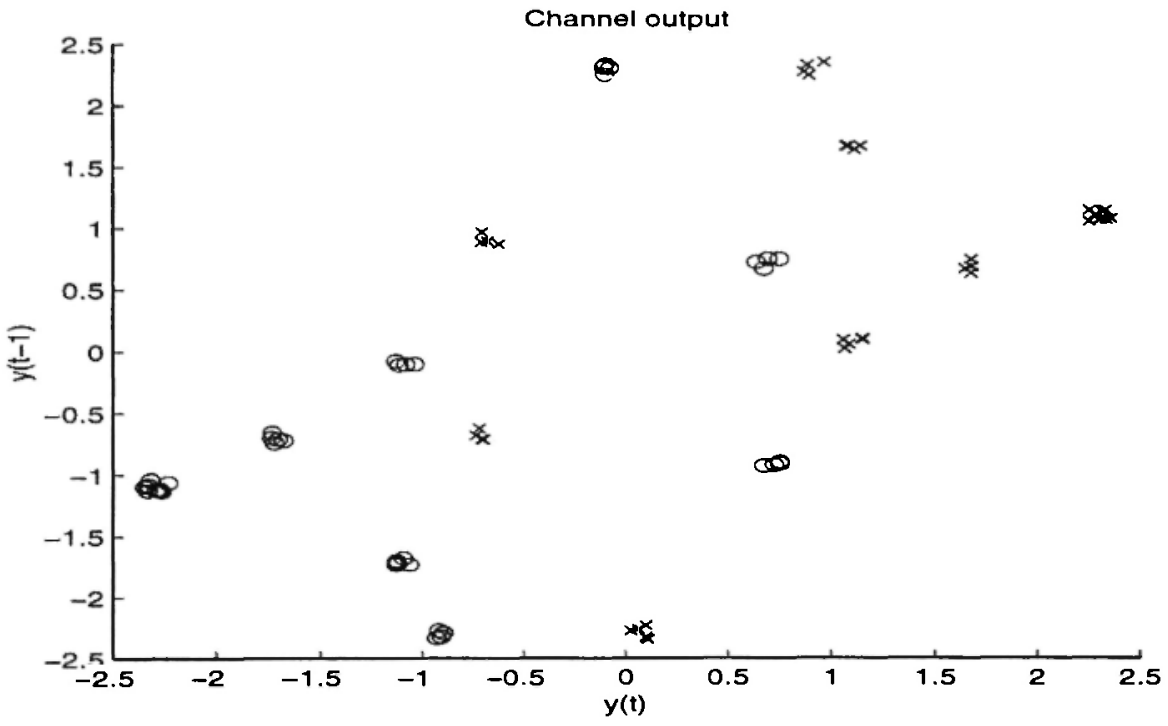

Fig. 2: Two-dimensional scatter plot of channel outputs. Symbol ' $x$ ' equals 1 and symbol 'o' equals -1 .

channel output points form several clusters, each indicating the true bit value that was transmitted. Now, the equalizer's task is to separate the channel output points that correspond to 1 from the points that correspond to -1 . In the case presented here, it is clearly visible that the clusters are not linearly separable. Therefore. it is expected that a conventional linear equalizer will not be able to give adequate performance. On the contrary, we expect that some nonlinearity must be added to the equalization process to be able to perform good equalization.

\subsection{Equalization techniques}

Conventionally, equalization methods can be divided into three types /11/.

1) The first method is based on the use of a linear filter with adjustable coefficients $/ 11 /$. 
2) A second equalization method is called decision-feedback equalization (DFE) $/ 15,16 \%$.

3) The third method is based on the maximum likelihood (ML) sequence estimation $/ 1,17,18 /$.

In recent years, new equalization techniques have been studied and found useful. For example, much research has been carried out on neuralnetwork-based equalizers, which have been found to perform the equalization task very well. Several types of neural network equalizers (with several types of training algorithms (for example /19-23/) have been studied, including, for example, multilayer perceptron (MLP) networks /5$7 /$, radial basis function (RBF) networks $/ 8,9,24,25 /$, and self-organizing maps (SOM) /26-28/. Clustering techniques for equalization have also been studied $/ 29,30 /$. Neural networks can be applied to DFE-based structures $17,9,31,32 /$ as well as to implement an optimal Bayesian Transversal Equalizer (TE) $/ 9 /$.

In this paper, we have studied three different equalization techniques. The first is a conventional linear equalizer. which is used as a comparative method for the others. The second technique is a neural network equalizer, which in our case was chosen to be a multilayer perceptron network. An adaptive clustering method, which has similarities to equalization with RBF networks and SOM, is the third equalization technique studied. The bit error rates achieved with these three equalization methods are compared with those achieved with an optimal Bayesian equalizer, and computational load of the methods is also studied.

\subsection{Application model}

In this paper, we use the following adaptive equalizer model. First, data is transmitted in bursts consisting of training sequence and data sequence (see Fig. 3). The equalizer uses a known comprehensive training sequence, which it receives repeatedly after a certain amount of time, to adjust the equalizer coefficients. Now the adjustment of the coefficients can be done after the whole training sequence is received. We call this adjustment of the equalizer coefficients training of the equalizer. Figure 4 presents a picture of this type of adaptive equalizer. There the channel output is fed into the equalizer. The equalizer output is then taken to a slicer, which makes 


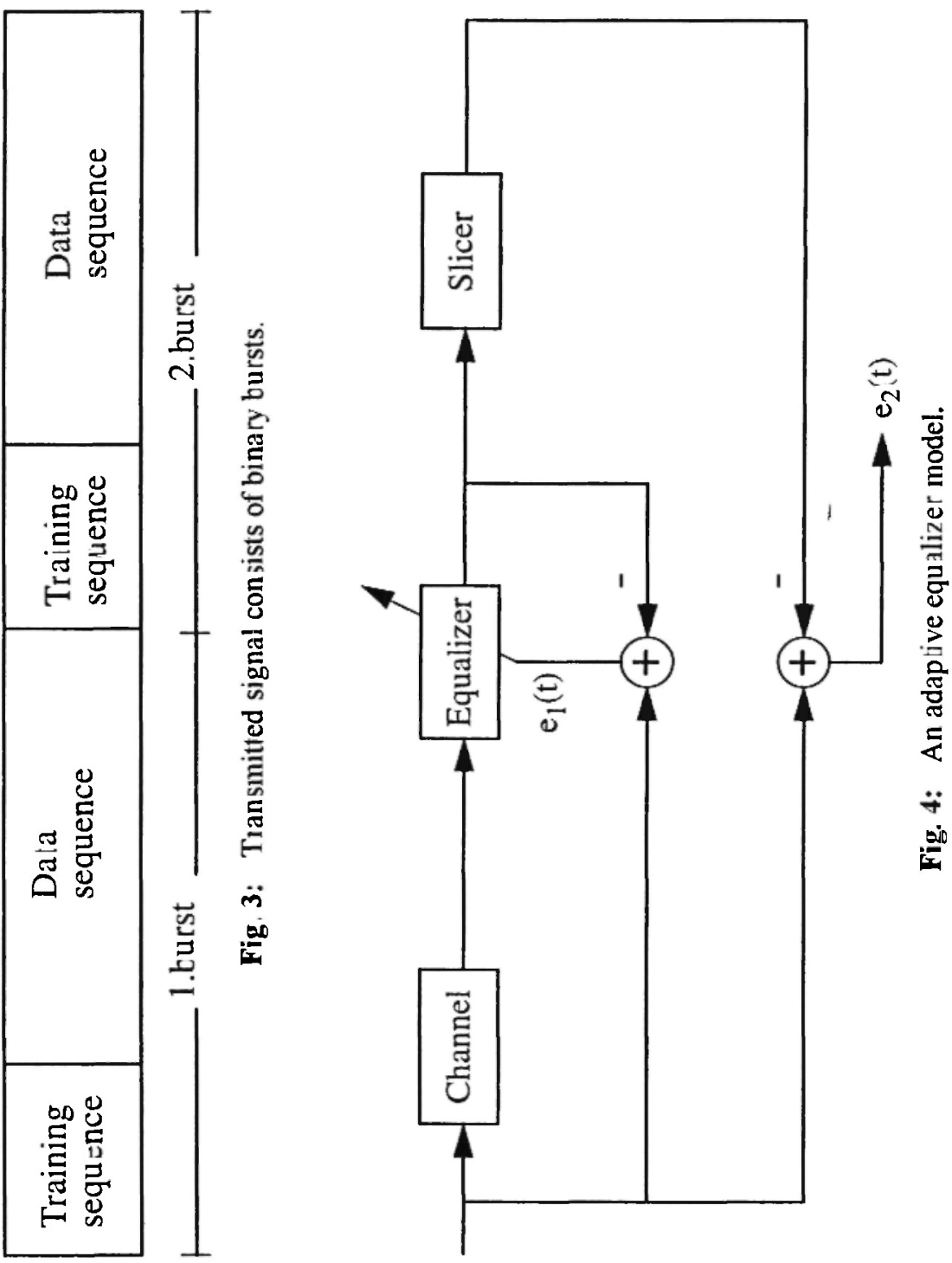


decisions of the equalizer output. The error signal of the equalizer, $e_{1}(t)$, is formed by subtracting the equalizer output from the known training signal. Now the equalizer coefficients are adjusted when the entire training sequence has been received. After the training sequence, the data sequence must be equalized. The data can now be equalized without adaptation with the trained equalizer. When the next training sequence is transmitted and received, the equalizer is re-trained. Thus, by adaptivity we mean here that the equalizer is burst adaptive.

The performance of the simulated equalization methods is studied as follows. First the equalizer is trained. Training is done using the training sequence attached to the transmitted burst and must be done separately for each transmitted burst. When the whole training sequence has been processed through and the equalizer is thus adapted, the entire training sequence is fed again to the adapted equalizer. This time, no adaptation is performed, but the training sequence is simply equalized with the adapted equalizer and hard-limited with the slicer to find the error $e_{2}(t)$ (see Fig. 4). This error is the final training error of the equalizer and is formed by subtracting the slicer output from the transmitted training sequence. The bit error rate for the training sequence is now formed as follows:

$$
\text { bertr }=\frac{1}{2}\left(\frac{1}{n} \sum_{t=1}^{n} e_{2}(t)\right),
$$

in which $n$ is the number of training samples and bertr tells us the bit error rate for the training sequence when it is equalized with the trained equalizer. The binary data burst is assumed to have values 1 or -1 .

After the equalizer is trained. we use it to equalize the data sequence. In this case, the channel output, which is now the data sequence. is fed to the trained equalizer. The equalizer output is then fed to the slicer. which again produces $[1,-1]$ output signal. The slicer output is then subtracted from the transmitted signal to find the error $e_{2}(t)$ for the data sequence. The bit error rate for the data sequence is calculated as in Eq. (2), but now $n$ is the number of data samples. This bit error rate is the most important characteristic of the system because it describes how many bit errors remain in the actual data sequence after the equalization. Of course. the goal is that no bit errors remain. 


\section{Equalization Methods}

In this section we shall describe three different equalization methods. The first equalization method described is a linear equalizer, in which the equalizer coefficients are adjusted in the least mean square sense. The second method is a multilayer perceptron network, and finally the third one is an adaptive clustering method.

\subsection{Linear equalizer}

The output of the linear equalizer can be given by

$$
\underline{z}=Y \underline{w},
$$

where $\underline{z}$ is the $N^{*} 1$ vector consisting of the optimal equalizer outputs, $Y$ is the $N^{*}(L+1)$ matrix including the channel outputs, and $\underline{w}$ is the $(L+1)^{*} 1$ vector including the weight values, $\underline{w}=\left[w_{0} w_{l}, \ldots, w_{L}\right]^{T}$.

Here the optimal weight values can be found by using linear regression. The goal is to minimize the error between the original transmitted signal and the equalizer output as given in the following equation:

$$
E(t)=\frac{1}{2} \sum_{t=1}^{N}(a(t)-z(t))^{2}
$$

where $E(t)$ is the sum square error between the transmitted signal and the equalizer output, $N$ is the number of training patterns, $a(t)$ is the original transmitted signal, and $z(t)$ is the equalizer output for $a(t)$. The following linear regression method results in globally optimal values for the weights in the sense that the error function gets minimized. By using matrix notation the error function can be written as

$$
E=\frac{1}{2}\left(\underline{a}-Y_{\underline{w}}\right)^{T}(\underline{a}-Y \underline{w})
$$

where $\underline{a}$ is $N^{*} 1$ vector including the desired outputs. $\underline{a}=\left[a_{1} a_{2} \ldots \ldots a_{N}\right]^{T}$. The input value for the bias term $w_{0}$ is assumed to be one. To minimize the error $E$, we must have 


$$
\nabla E=-Y^{T} \underline{a}+Y^{T} Y \underline{w}=0
$$

The matrix $Y^{T} Y$ should be positively definite. Then the weights $\underline{w}$ can be solved from equation

$$
\underline{w}=\left(Y^{T} Y\right)^{-1} Y^{T} \underline{a} .
$$

The term $\gamma^{T} Y^{-1} Y^{T}$ is called the pseudoinverse of matrix $Y$.

The matrix operations described above do not require a lot of memory. Matrix $Y$ can be presented as follows:

$$
Y=\left[\begin{array}{c}
\underline{y}_{1} \\
\cdots \\
\underline{y}_{N}
\end{array}\right],
$$

where $y_{i}$ is the $1^{*}(L+1)$ vector. Now

$$
Y^{T} Y=\sum_{i=1}^{N} \underline{y}_{t} \underline{y}_{i}^{T}
$$

which is autocorrelation matrix of $y_{1}$. Similarly

$$
Y^{T} \underline{a}=\sum_{i=1}^{N} \underline{y}_{i} a_{i}
$$

which is the cross-correlation vector between $y_{i}$ and $a_{i}$.

\subsection{Multilayer perceptron network}

The multilayer perceptron network consists of processing units, also called neurons, which perform some simple mathematical operations, and are typically arranged in layers connected to each other $/ 20 \%$. The output of one MLP processing unit can be described by the following equation: 


$$
o_{j}(t)=f\left(w_{0 j}+\sum_{i=1}^{\vec{r}} w_{i j} y(t-i+1)\right),
$$

where $w_{y}$ are weights representing the strength of the connections, $y(t-i+1)$ is the ith input, and $f$ is an activation function. Parameter $w_{0 j}$ represents the bias term. The inputs $v(t)$ are coming from other processing units or they are the network inputs. The input signals are multiplied by the weights and the results are summed up to form an activation signal. The activation signal is fed into the activation function $f$. The activation function either activates or deactivates the output, depending on the magnitude of activation signal. Several activation functions are commonly used with MLPs, for instance, the step function and piecewise linear function. If the network training is done by using gradient based algorithms, which require that the first derivative of the activation function is continuous, then the sigmoidal functions are often used. Examples of sigmoidal functions are the logistic function and hyperbolic tangent (tanh) function.

In our case, we have used one hidden layer in our network, so the network output is

$$
z_{k}(t)=f_{o}\left(v_{0 k}+\sum_{j=1}^{q} v_{i k} f_{h}\left(w_{0, i}+\sum_{i=1}^{p} w_{i j} v(t-i+1)\right)\right)
$$

where $z_{k}$ is the output of the $k$ th output unit, $v_{j k}$ and $w_{j j}$ are the network weights, $p$ is the number of network inputs, $q$ is the number of hidden units, $f_{h}$ is the activation function of the hidden layer, and $f_{o}$ is the activation function of the output layer. The training of the MLP network is done in a supervised manner. We know the desired output $a(t)$ for certain input $y(t)$. In training. the weights are adjusted in such a way that the difference between the network output $z(t)$ and the desired output $a(t)$ is minimized. Usually this is done by minimizing the cost function given in Eq. (4). The minimization of the cost function is usually done by gradient descent methods. In our case, the training was done by using the RPROP algorithm /33/. RPROP is a local adaptive learning scheme in which the basic principle is to eliminate the harmful influence of the size of the partial derivative on the weight step. The 
algorithm goes as follows. An individual update-value $\Delta(t)$ for each weight is introduced. This update-value determines the size of the weight-update. The update-value is adapted from the following equation:

$$
\Delta(t)=\left\{\begin{array}{cc}
\eta^{p} \Delta(t-1) & \text { if }\left(\frac{\partial E(t-1)}{\partial 0} \times \frac{\partial E(t)}{\partial 0}>0\right) \\
\eta^{n} \Delta(t-1) & \text { if }\left(\frac{\partial E(t-1)}{\partial 0} \times \frac{\partial E(t)}{\partial 0}<0\right) \\
\Delta(t-1) & \text { else }
\end{array}\right.
$$

where $0<\eta^{n}<1<\eta^{p}$ and $\theta$ represents the network weights $w$ and $v$. Eq. (12) means that every time the derivative of the corresponding weight $\theta$ changes its sign, which indicates that the last update was too big and the algorithm has jumped over a local minimum, the update-value $\Delta(t)$ is decreased by the factor $\eta^{n}$. If the derivative retains its sign, the update-value is slightly increased to accelerate convergence. When the update-value for each weight is adapted, the weight-update itself follows a very simple rule. If the derivative is positive, the weight is decreased by its update-value. If the derivative is negative, the update-value is added (see Eqs. (13) and (14)).

$$
\begin{aligned}
& \Delta(t)=:\left\{\begin{array}{lc}
-\Delta(t) & \text { if }\left(\frac{\partial E(t)}{\partial 0}>0\right) \\
-\Delta(t) & \text { if }\left(\frac{\partial E(t)}{\partial 0}>0\right) \\
0 & \text { else }
\end{array}\right. \\
& \theta(l+1)=\theta(l)+\Delta \theta(l) .
\end{aligned}
$$

There is one exception to the rule above. In case of a change in sign, no adaptation should occur in the succeeding learning step. In practice this can be achieved by setting

$$
\frac{\partial E(t-1)}{\partial \theta}=0
$$

in Eq. (12). 
The update-values and the weights are changed every time the whole training set has been presented once to the network. This is called learning by epoch, where an epoch means the period in which every pattern of the training set is presented once. The advantage of RPROP is that the adaptation effort is not blurred by unforeseeable gradient behavior because only the sign of the partial derivative is used to perform both learning and adaptation. In this way fast convergence can be achieved. To reduce the number of freely adjustable parameters, the increase and decrease values are set to fixed values. A good value for the decrease factor $\eta^{n}$ is $\mathbf{0 . 5}$. For the increase factor, $\eta^{p}=1.2$ was found out to be a sufficient value in $/ 33 /$.

\subsection{Adaptive clustering}

As discussed earlier, equalization can be seen as a classification problem. Clustering methods are often used for classification problems /34/. In clustering we classify data to several groups, also called clusters. Here we shall present an adaptive clustering method for equalization. The basic idea is first to find adaptively the cluster centers from a known training signal and then classify the data bits according to these clusters. The training signal is needed because the output of the communication channel is usually unknown. Therefore. we do not know where the clusters will appear.

The adaptive clustering algorithm goes as follows. The transmitted signal $a$ is a binary burst consisting of training-sequence and data-sequence taking values of 1 or -1 . A sample vector $\underline{v}$ is formed from the received signal as follows:

$$
\underline{y}=\left[\begin{array}{c}
y(t) \\
y(t-1) \\
y(t-k)
\end{array}\right],
$$

in which $k+1$ is the number of inputs. The known training sequence is first used to find the cluster centers. If the transmitted training bit is 1 and it is the first 1-bit, it forms the first 1-cluster center $c p_{1}$. which is now the same vector $v$ that was given as an input at time $t$. The same goes for the first transmitted -1-bit, meaning that if the transmitted training bit is -1 and it is the first -1 -bit, it forms the first -1 -cluster center $\underline{c} n_{1}$. Otherwise the received bits are processed through the following procedure: 
1. At the time instant $t$, it is checked whether the transmitted training bit $a(t)$ was 1 or -1 .

2. If $a(t)$ was 1 , then we find the minimum distance between the received sample vector $\underline{v}$ and all the 1-cluster centers by

$$
d_{m ! n}=\min _{i}\{\|v-\underline{c p},\|\}
$$

where $d_{m ! n}$ is the minimum distance between the received sample vector $y$ and 1 -cluster centres $\underline{c p}$. The norm is assumed to be Euclidean.

3. The smallest of the distances $d_{m ! n}$ is then compared to a threshold value $d_{i h}$.

4. If the smallest distance $d_{m ! n}$ to a cluster center $\underline{c p} \underline{k}_{k}$ is smaller than the threshold value, then the received sample vector $\underline{y}$ is classified to the cluster center $\underline{c p_{k}}$. The location of the cluster center $\underline{c p_{n}}$ is updated by the weighted mean as follows:

$$
\underline{c p}_{k}=\frac{\underline{c p} \times \underline{\underline{p}}_{k}+\underline{\underline{v}}}{n \underline{c p}_{k}+1},
$$

in which $\underline{c p}_{k}$ is the location of the cluster center, $n \underline{c p} \underline{w}_{k}$ is the number of samples classified to $\underline{c p}_{k}$, and $\underline{v}$ is the received sample vector. The number of samples that the cluster center $c \underline{p}_{k}$ contains is also updated. If the smallest distance $d_{m i n}$ to a cluster center $\underline{c p}_{k}$ is larger than the threshold value, then the sample vector $\underline{v}$ forms a new 1 -cluster center.

5. If $y(t)$ was the last received training sample, then quit, otherwise set $t=t+1$ and continue at step 1 .

If the transmitted training bit at step 2 is -1 , then the same actions are done with the -1 -clusters.

When the training sequence has been processed through and the cluster centers are found, the equalization of the data sequence is simple. The Euclidean distances from the received sample to all cluster centers are calculated. and the sample is classified to the cluster to which the distance is smallest according to 


$$
m=\arg , m m\left\{\underline{L} \underline{\underline{y}-c_{i}} \|\right\}
$$

where $m$ is the index of the cluster center to which the sample is classified, and $c$, represents $t$ th cluster center. The binary value of the received sample is now defined by the cluster to which it is classified.

\section{Simulation Results}

The transmitted signal considered is a burst of bits, which has some features that are similar to a GSM burst /2,3/ (see Fig. 3 in section 2.3.). The burst consists of 142 bits. The first 26 bits of the burst are called training bits. These bits are used to train the equalizers to compensate for the channel influences as much as possible. The training sequence is a fixed sequence of bits, which is known at the receiver end. The last 116 bits, which are called data bits, contain the information being sent. These are the bits that need to be equalized. Thus, they are not known at the receiver end. The transmitted data are assumed to be binary, taking values of either 1 or -1 . Before being transmitted to the channel, the burst is oversampled so that for every bit, three samples are taken. Therefore, the actual number of bits in one transmitted burst is 441 . Simulations were made separately for fixed and altering channels.

\subsection{Fixed channel}

The impulse response of the channel was set to have a value $\underline{h}=(h(0)$, $h(1), \ldots, h(4))=\left(\begin{array}{lll}0.5 & -0.30 .6-0.7-0.8\end{array}\right)$. To take a closer look at how the three equalization methods perform, we shall first observe an example case, in which the noise variance is given value $\sigma^{2}=0.001$. In this example case, the channel output is as presented in Fig. 2 (in section 2.1.). The goal of the equalization is now to be able to separate the clusters representing trānsmitted 1-bit from the clusters representing transmitted -1-bit.

The linear equalizer was used as described in section 3.1. The number of weight values used in simulations varied between $1, \ldots, 10$. The minimum bit error rate obtained for the data sequence was 0.3678 with 9 weight values. 
a)

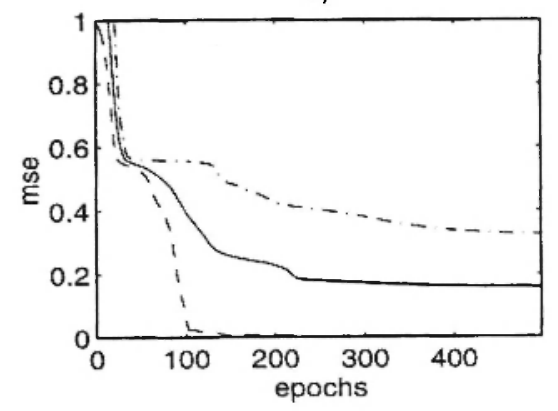

b)

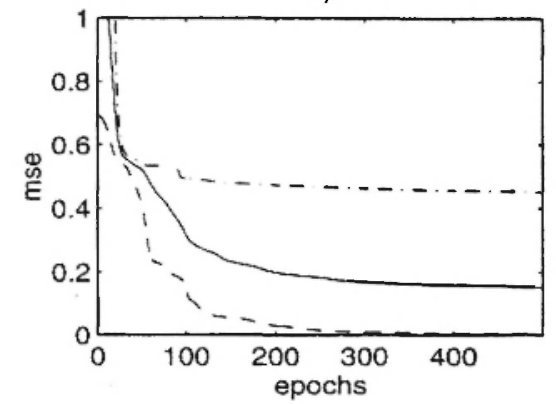

c)

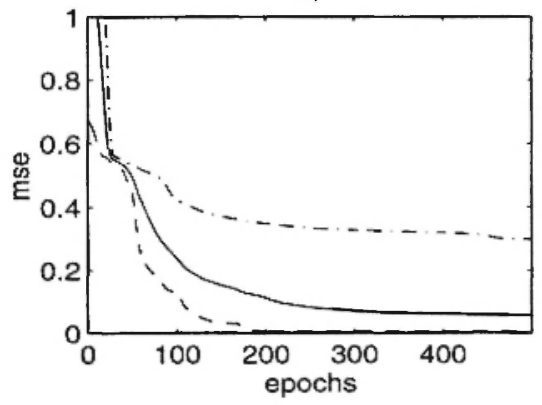

d)

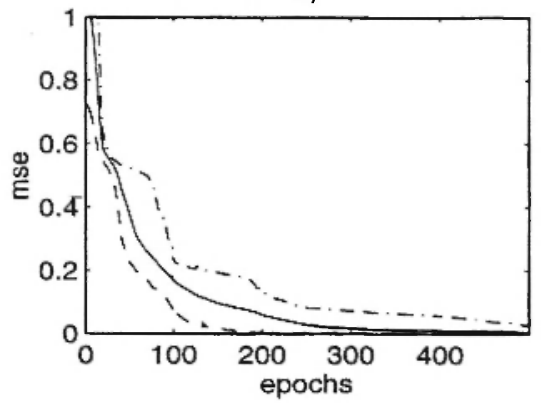

Fig. 5: Training curves for MLP networks with (a) three, (b) five, (c) seven, and (d) nine hidden units. - , average mean square error (MSE), - - - maximum MSE, - - , minimum MSE.

This is obviously too large, meaning that the linear equalizer is not able to perform good equalization in our example case. This was already expected because the clusters in Fig. 2 are not linearly separable.

According to our empirical simulations, the best number of MLP network inputs was found out to be two. The output layer had one processing unit, and it was set to be linear, and the activation function in the hidden 
units was chosen to be the hyperbolic tangent (tanh) function. The number of hidden units varied between $1, \ldots, 10$. The network was trained with RPROP-algorithm for 500 iterations or epochs. In this application a larger number of epochs would be computationally too exhaustive.

For every network the simulations were repeated 10 times because of random initialization of the network weights (interval $-0.5, \ldots, 0.5$ ). Figure 5 shows the learning curves for four networks with two inputs differing in the number of hidden units. As can be seen, the training is very sensitive to the random weight initialization. With fewer number of hidden units, it is more probable that the network does not converge to a good solution because poor initialization has caused some of the hidden units to be useless. Of course, all the units would be effectively needed to achieve the desired mapping. With a larger number of hidden units, there can be unnecessary units, if the other units can handle the problem at hand. Nevertheless, the unnecessary hidden units also cause a lot of unnecessary computation.

Finally, we also simulated the adaptive clustering method, as described in section 3.3 for the example case. The critical factor in the adaptive clustering method is the selection of the threshold value $d_{t h}$. If the threshold value is chosen to be too big, the adaptive clustering method will not be able to perform equalization without errors because there are not enough cluster centers in which to classify the data bits, and thus some of the bits are wrongly classified. If, however, the threshold value is chosen well, the adaptive clustering method achieves very good results. In our simulations, it achieved BER equal to zero when the threshold value was $0<d_{t h}<0.8$.

Next we simulated the three equalization methods with different signal to noise ratios (SNR). The channel impulse response was kept the same and SNR was given values between $3, \ldots, 30 \mathrm{~dB}$. The simulated linear equalizer was set to have ten weight values. As an MLP network, we simulated a network with two inputs, nine hidden neurons and a single output unit. The activation functions were also kept the same. The adaptive clustering method had also two inputs. For comparison, also the results obtained with an optimal Bayesian equalizer /35/ are presented. Figure 6 shows the bit error rates for the data sequence achieved with each equalization method as a function of SNR. It can be seen from Fig. 6 that the linear equalizer (dotted line) is not useful in this kind of a channel. Since the classification problem considered here is not linearly separable, the performance of the 


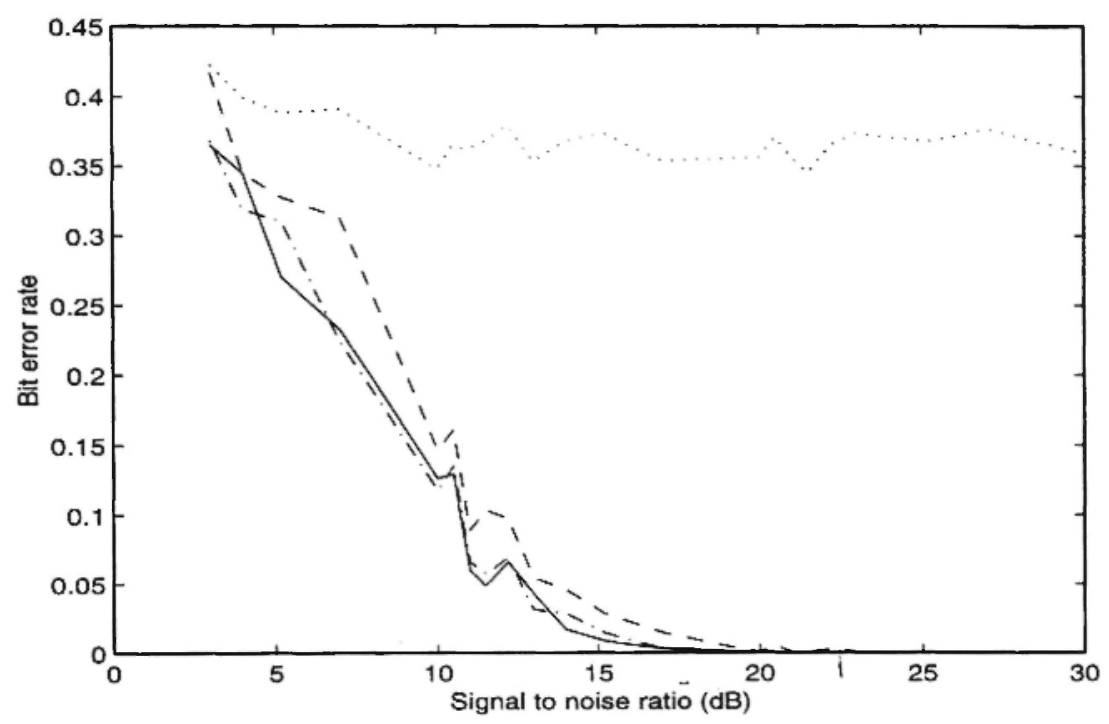

Fig. 6: Bit error rates for the data sequence as a function of signal to noise ratio. -, optimal Bayesian equalizer; - -. MLP network; - - ', adaptive clustering; . . ', linear equalizer.

linear equalizer does not improve much when the signal to noise ratio increases. Both the MLP network (dashed line) and adaptive clustering method (dashdotted line) perform very well in this channel. Their performance improves rapidly when signal to noise ratio exceeds $5 \mathrm{~dB}$. In some cases the adaptive clustering method achieves even smaller BER than the optimal Bayesian equalizer (solid line). The adaptive clustering method achieves bit error rate equal to zero when the signal to noise ratio exceeds 20 $\mathrm{dB}$. The MLP network also achieves a bit error rate equal to zero when the signal to noise ratio exceeds $20 \mathrm{~dB}$. If, however, the network weights are initialized poorly, the bit error rate can be larger than zero, even for greater value of SNR. 
a)

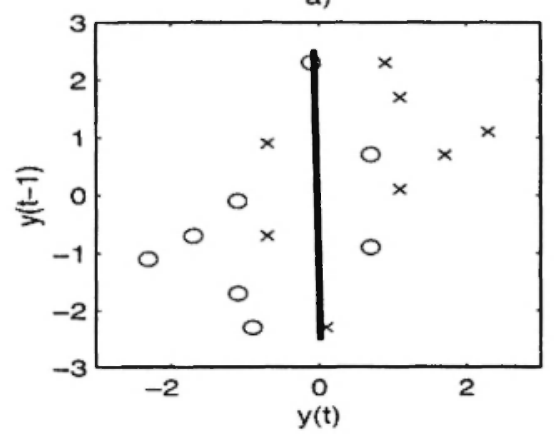

c)

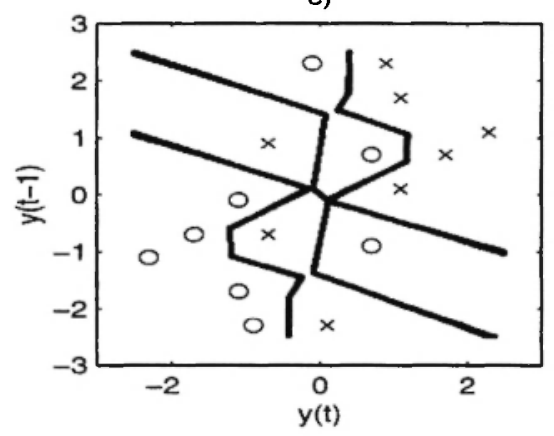

b)

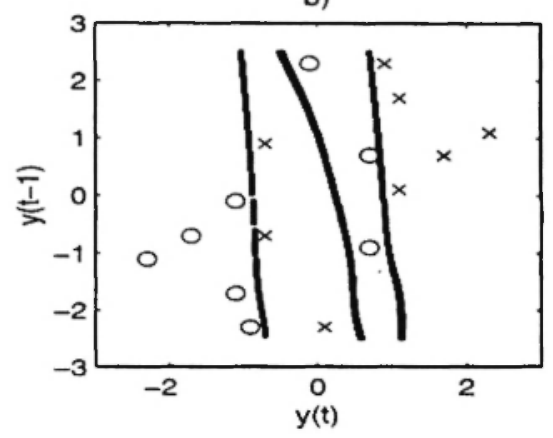

d)

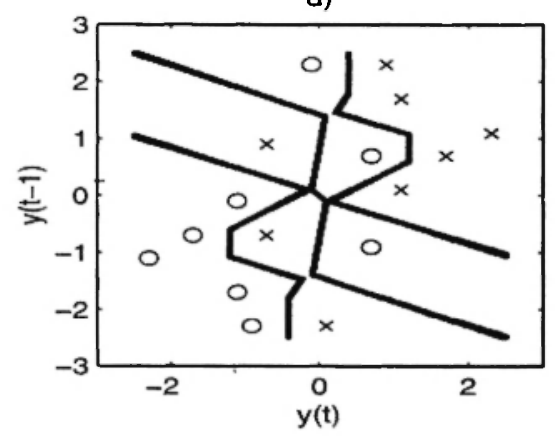

Fig. 7: Decision boundaries for (a) linear equalizer, (b) MLP network, (c) adaptive clustering, and (d) optimal Bayesian equalizer, when SNR is $30 \mathrm{~dB}$.

Figure 7 depicts the decision boundaries for each equalization method when SNR is $30 \mathrm{~dB}$. Here the linear equalizer was used with only two inputs like the other methods. In Fig. 7, the optimal cluster centers have been marked with symbols ' $x$ ' if it corresponds to a 1-cluster center and ' 0 ' if it corresponds to a -1-cluster center. As can be seen, the linear equalizer does not perform well because the cluster centers are not linearly separable. The MLP network and the adaptive clustering method apply nonlinear decision boundaries and therefore achieve better results. 
Figure 8 depicts what happens when the SNR is small. Again the linear equalizer tries to solve the equalization problem with a linear decision boundary. The MLP network and the adaptive clustering method create highly nonlinear decision boundaries. In this case, however, the noise is so powerful that neither method can perform the equalization without errors because high noise causes the samples of different cluster centers to overlap and thus to be classified incorrectly.

a)

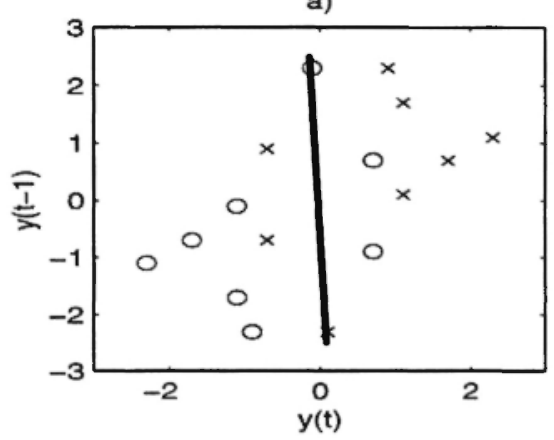

c)

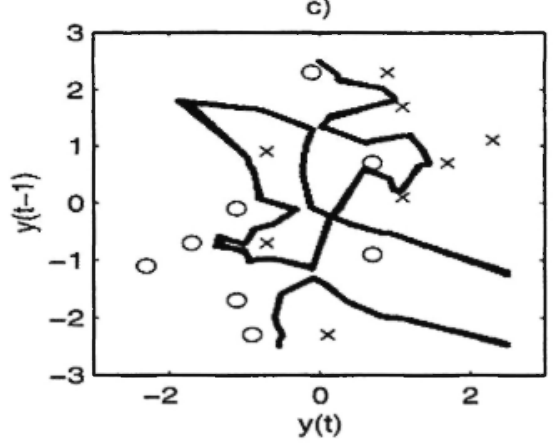

b)

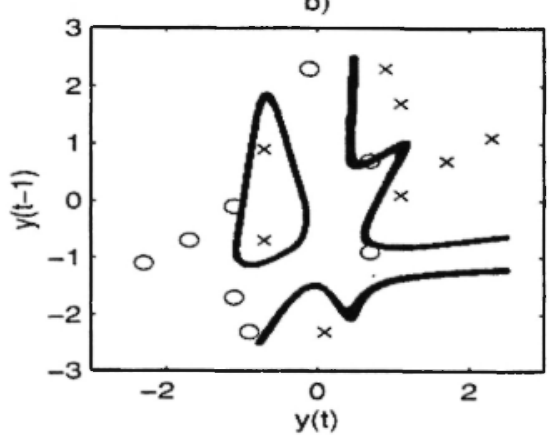

d)

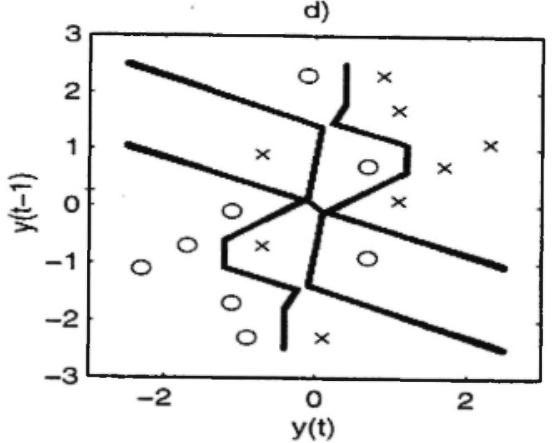

Fig. 8: Decision boundaries for (a) linear equalizer, (b) MLP network, (c) adaptive clustering, and (d) optimal Bayesian equalizer, when SNR is $10 \mathrm{~dB}$. 


\subsection{Computational load}

Another important factor, especially in mobile systems, is the computational load of the system. The computational load was measured by observing the number of floating point operations used for the training of each equalization method. For the linear equalizer, we calculated the number of floating point operations required to find the optimal weight values. For the MLP network, we measured the number of floating point operations required to train the network with RPROP algorithm. For the adaptive clustering method, we measured the number of floating point operations required to find the cluster centers from the training sequence. Both the MLP network and the adaptive clustering method were used with two inputs.

Table 1 presents the number of floating point operations (marked with $f)$ required for the linear equalizer as a function of number of weight values (marked with $L$ ). Table 2 presents the number of floating point operations required for the MLP network as a function of number of hidden neurons (marked with $q$ ). Here $l$ is divided by 500 to find the number of floating point operations required to train the network with the training sequence once (the network was trained for 500 epochs). In Table 3, the number of

\section{Table 1}

The number of floating point operations $f$ for linear equalizer as a function of number of weight values $L$.

\begin{tabular}{|l|c|c|c|c|c|c|c|c|c|c|}
\hline$L$ & 1 & 2 & 3 & 4 & 5 & 6 & 7 & 8 & 9 & 10 \\
\hline$n$ & 1593 & 3368 & 5810 & 8925 & 12735 & 17248 & 22476 & 28431 & 35125 & 42570 \\
\hline
\end{tabular}

\section{Table 2}

The number of floating point operations $f$ for MLP network as a function of number of hidden neurons $q$.

\begin{tabular}{|c|c|c|c|c|c|c|c|c|c|c|}
\hline$q$ & 1 & 2 & 3 & 4 & 5 & 6 & 7 & 8 & 9 & 10 \\
\hline$\pi / 500$ & 2596 & 4642 & 6611 & 8579 & 10547 & 12516 & 14484 & 16454 & 18421 & 20389 \\
\hline
\end{tabular}


Table 3

The number of floating point operations $f$ for adaptive clustering method as a function of threshold value $d_{t h}$

\begin{tabular}{|c|c|c|c|c|c|c|c|c|c|}
\hline$d_{\text {th }}$ & 0.0 & 0.1 & 0.2 & 0.3 & 0.4 & 0.5 & 0.6 & 0.7 & 0.8 \\
\hline$n c(l)$ & 39 & 10 & $\mathbf{8}$ & $\mathbf{8}$ & $\mathbf{8}$ & $\mathbf{8}$ & $\mathbf{8}$ & 7 & 6 \\
\hline$n c(-l)$ & 39 & $\mathbf{8}$ & $\mathbf{8}$ & $\mathbf{8}$ & $\mathbf{8}$ & $\mathbf{8}$ & $\mathbf{8}$ & 6 & 6 \\
\hline$f l$ & 19730 & $\mathbf{8 3 3 8}$ & 7661 & 7661 & 7661 & 7661 & 7661 & 6470 & 6099 \\
\hline
\end{tabular}

floating point operations required for the adaptive clustering method are presented as a function of threshold value $d_{t h}$. With adaptive clustering, the number of floating point operations required depends on how many cluster centers we have. Therefore, this has also been presented in Table 3 (the number of 1-cluster centers is marked with $n c(l)$, and the number of -1 -cluster centers is marked with $n c(-l)$ ).

As can be seen from Tables 1. 2, and 3, a linear equalizer with one weight value needs the smallest amount of floating point operations. However with this kind of equalizer, the simulation results are very poor, that is, the bit error rate is large. To improve the results for the linear equalizer, we must use more than one weight value. This, of course, increases the number of floating point operations required, as can be seen in Table 1. Still, when using 10 weight values, the simulation results are poor when compared with the other two equalization methods considered (see Fig. 6). With the adaptive clustering method. we achieved good results and the amount of floating point operations required was quite small. With the MLP network, we could achieve results that were almost as good in terms of bit error rate as those achieved with the adaptive clustering method, but the number of floating point operations required is considerably larger because the network must be trained several times with the training sequence (in this case max. 500 iterations). The computation in MLP networks can be done in a parallel manner, however, and we can thus decrease the actual time spent in computation. Also, if some efficient weight initialization method were used $/ 36,37 /$, then the network could converge faster, and thus, the number of training epochs could be made smaller. This, of course, also decreases the number of floating point operations required for the training. 


\subsection{Altering channel}

We have also simulated an altering channel by transmitting several bursts consecutively in two cases differing in noise. Now the channel impulse response was different for every transmitted burst. The channel impulse response $\underline{h}=(h(0), h(1), \ldots, h(4))$ was given uniformly distributed, randomly chosen values between $-1, \ldots, 1$ for every transmitted burst. The noise distribution in two different cases can be found from the histograms presented in Fig. 9.

The simulated linear equalizer was set to have ten weight values. The MLP network was set to have two inputs and nine hidden units and a single output unit. Here we simulated two kinds of MLP networks. The first one used as cost fimction the mean square error (MSE) given in Eq. (4). The second MLP network was trained by maximizing the relative entropy criterion $/ 19,38,39 /$

$$
H=\sum_{t=1}^{n}\left[(1-\tilde{u}(i))_{i n} \frac{1-a(t)}{1-z(t)}+(1+\bar{u}(t))_{\operatorname{lin}} \frac{1+a(t)}{1+z(t)}\right] \text {, }
$$
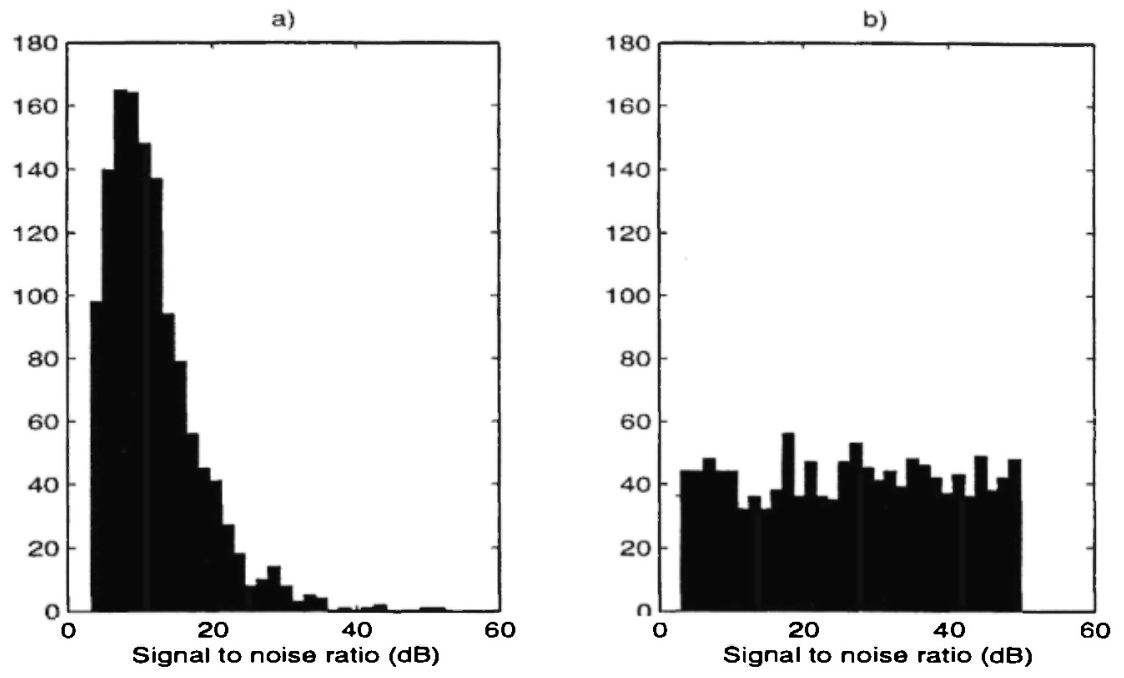

Fig. 9: Histograms of noise distributions when simulating the altering channel 
in which $a(t)$ is the desired output, $z(t)$ is the network output, and $n$ is the number of training samples. When using relative entropy. we also need to use suitable activation function in the processing unit in the output layer. Here we have used the hyperbolic tangent function. The adaptive clustering method was set to have two inputs. and the threshold value $d_{\text {th }}$ was chosen to be 0.1 . Also, the results for the optimal Bayesian equalizer are presented.

\section{Table 4}

Simulation results with altering channel where noise is distributed as depicted in Fig. 9

\begin{tabular}{|l|c|c|}
\hline Method & bertr $_{a v}$ & berdata $_{a v}$ \\
\hline Linear equalizer & 0.1340 & 0.2885 \\
\hline MLP1 & 0.0775 & 0.2034 \\
\hline MLP2 & 0.0656 & 0.2025 \\
\hline Adaptive clustering & 0.0021 & 0.2001 \\
\hline Optimal Bayesian & 0 & 0.1613 \\
\hline
\end{tabular}

Table 4 shows the average bit error rates for the training sequence (bertr) and the data sequence (berdata) that we obtained with each method when noise is distributed as depicted in Fig. 9a. The simulations were made so that 1270 consecutive bursts were transmitted altogether. This number of bursts corresponds to the amount of bursts required during about two seconds of transmission in the GSM system. In Table 4, MLPI denotes the MLP network where the cost function was the MSE. MLP2 denotes the MLP network where the relative entropy criterion was used.

From the results, it can be seen that the bit error rates for each equalization method are quite large because of the very powerful noise. The adaptive clustering method achieved better BERs than the linear equalizer and the MLP networks. Nevertheless, the difference in BERs between the adaptive clustering method and the MLP networks is very small. In this case, the network that used the relative entropy criterion was slightly better than that using the MSE criterion. The performance of the MLP network could be improved if it were provided with good initial weight values. Again, the performance of the linear equalizer was found to be clearly the worst. Figure 
a)

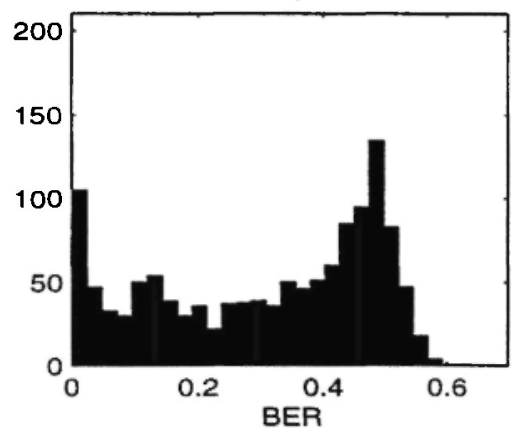

c)

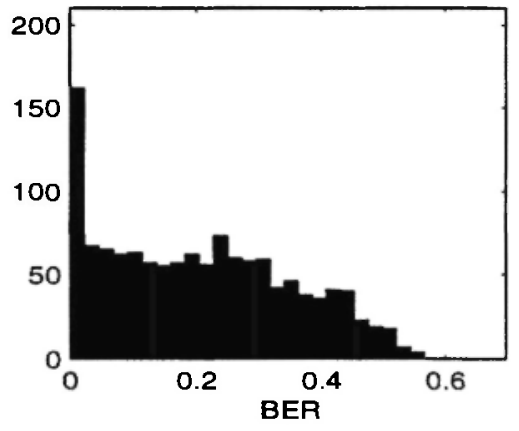

b)

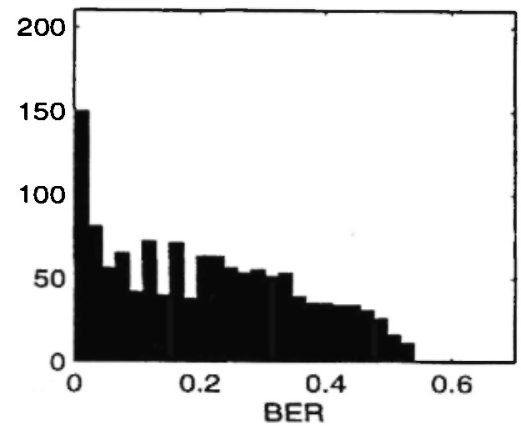

d)

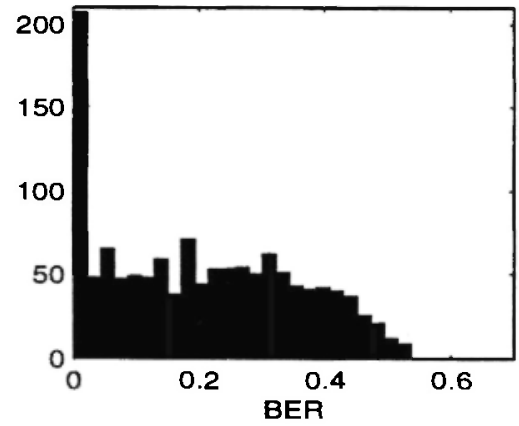

Fig. 10: Histograms of the BERs achieved with (a) linear equalizer, (b) MLP1 network, (c) MLP2 network. and d) adaptive clustering method when noise is distributed as depicted in Fig. 9a.

Figure 10 shows a histogram of the bit error rates for the data sequence achieved with each equalization method. From the shapes of these histograms, it can be seen that with MLP networks and adaptive clustering. most of the equalized bursts had bit error rates that are equal to or very close to zero. With linear equalizer a lot of equalized bursts had very poor bit error rate. Secondly we simulated altering channel, where the noise is not as powerful as that in the first case. Figure 9b shows a histogram of the SNR values used in this simulation. It can be seen that the SNR values are now 
quite uniformly distributed between 3 and $50 \mathrm{~dB}$. The equalization methods were used in the same way as described earlier in this section. Table 5 shows the average bit error rates achieved with each method in this case. Now the bit error rates for MLP networks and the adaptive clustering method are less than half of the bit error rates obtained in the first altering channel case. Still, the adaptive clustering method resulted in smaller BER than MLP networks, but the difference was very small in this case too. Again the MLP network results could have been improved, if some good initial weight values were given to the network. The bit error rate for the linear equalizer was only slightly better than that in the first case.

\section{Table 5}

Simulation results with altering channel where noise is distributed as depicted in Fig. $9 \mathrm{~b}$

\begin{tabular}{|l|c|c|}
\hline Method & bertr $_{a v}$ & berdata $_{a v}$ \\
\hline Linear equalizer & 0.1195 & 0.2761 \\
\hline MLP1 & 0.0394 & 0.0967 \\
\hline MLP2 & 0.0334 & 0.0925 \\
\hline Adaptive clustering & 0.0030 & 0.0765 \\
\hline Optimal Bayesian & 0 & 0.0636 \\
\hline
\end{tabular}

Figure 11 presents the histograms of the achieved bit error rates with each equalization method. In this case, the difference between the linear equalizer and the three other methods is clear. With the adaptive clustering method, nearly 800 bursts and with MLP networks, about 700 bursts were equalized with a BER less than 0.02 . With the linear equalizer, this number of bursts was less than 250 . The linear equalizer also has a clearly larger number of bursts that are equalized with a poor BER than with the other equalization methods. 
a)

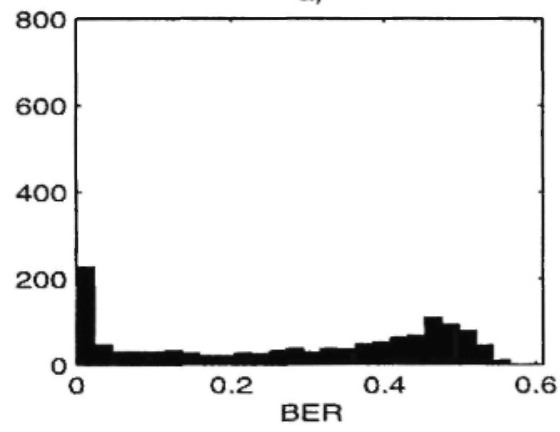

c)

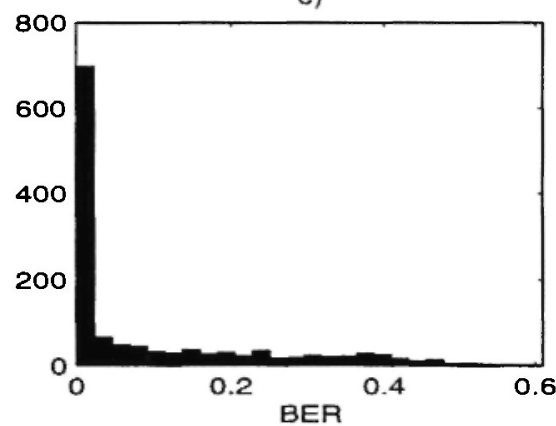

b)

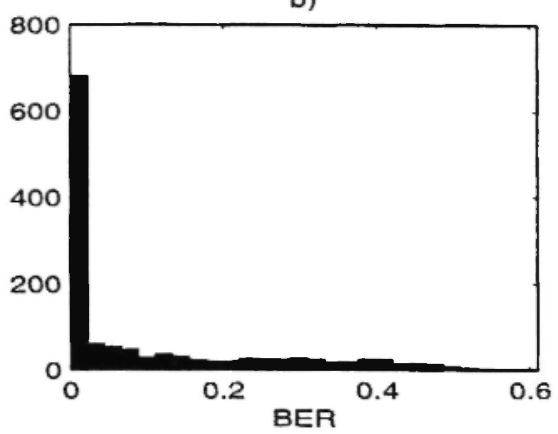

d)

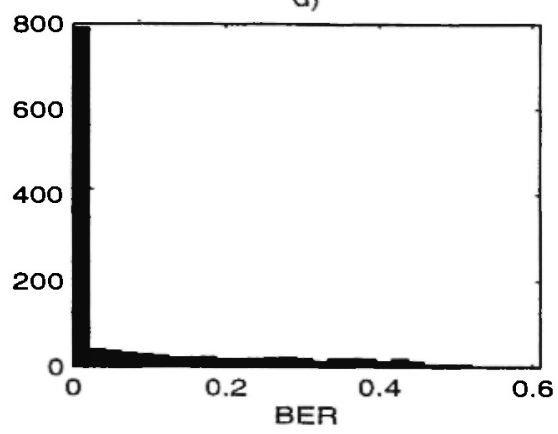

Fig. 11: Histograms of the BERs achieved with (a) linear equalizer, (b) MLP1 network, (c) MLP2 network, and (d) adaptive clustering method when noise is distributed as depicted in Fig. $9 \mathrm{~b}$.

\section{Conclusions}

By applying fixed and altering channels, it has been demonstrated that if intersymbol interference caused by channel is severe, a linear equalizer is not able to equalize the received signal properly. In such a case, we can use a nonlinear equalization process. A nonlinear neural network and adaptive clustering method were also used for equalization in the example case. It 
was shown that both outperformed the linear equalizer when bit error rates were considered. The disadvantage of the multilayer perceptron network was that the training did not always converge to an adequate solution. The main reason for this result was the random initialization of the network weights, which is commonly used before actual weight adjustment or training. To avoid this problem, a computationally efficient weight initialization method could be used.

When comparing the computational load of the equalization methods, it was found that linear equalizer with one weight value requires the smallest amount of floating point operations. The results obtained with this equalizer, however, were very poor. In practice, the linear equalizer needs more weight values and thus, also more floating point operations to improve the results. The adaptive clustering method also needed very few floating point operations, and the results obtained were much better than those with the linear equalizer and also slightly better than those with the MLP network. The MLP network clearly required the largest amount of floating point operations because the training sequence had to be processed through several times to train the network properly. The computation in the MLP network can be done in a parallel manner. however. which decreases the actual time used for computation. Also, the usage of a computationally efficient weight initialization method reduces the computational load, as the number of training epochs for the network can be decreased. This is very important especially in a mobile environment. where it is often not possible to use systems with a high computational load.

\section{References}

1. E.A. Lee and D.G. Messerschmitt. Digital Communication, Second ed., Kluwer Academic Publishers, 1994.

2. A. Kantsila, M. Lehtokangas, J. Saarinen, Adaptive equalization of binary data bursts, Proc. of IASTED International Conference on Signal and Image Processing (SIP'97), New Orleans, Louisiana, USA. Dec. 46, 1997, pp. 117-122.

3. M. Mouly, M-B. Pautet, The GSM System for Mobile Communications. Palaiseau: Mouly \& Pautet, 1992. 
4. K.A. Al-Mashouq. I.S. Reed, The use of neural nets to combine equalization with decoding for severe intersymbol interference channels, IEEE Trans. on Neural Networks, 5, 982-988 (1994).

5. S. Chen, G.J. Gibson. C.F.N. Cowan, P.M. Grant, Adaptive equalization of finite non-linear channels using multilayer perceptrons, Signal Processing, 20, 107-119 (1990).

6. G.J. Gibson, S. Siu, C.F.N. Cowan, The application of nonlinear structures to the reconstruction of binary signals, IEEE Trans. on Signal Processing, Vol. 39, No. 8, Aug. 1991, pp. 1109-1118.

7. M. Meyer and G. Pfeiffer, Multilayer perceptron based decision feedback equalisers for channels with intersymbol interference, IEE Proc. -1 , Vol. 140, No. 6, Dec. 1993, pp. 420-424.

8. S. Chen, B. Mulgrew, P.M Grant, A clustering technique for digital communications channel equalization using radial basis function networks, IEEE Trans. on Neural Networks, Vol. 4, No. 4, Jul. 1993, pp. 570-579.

9. B. Mulgrew, Applying radial basis functions, Signal Processing, Mar. 1996, pp. 50-65.

10. A.B. Carlson, Communication Sustems. Third edition, McGraw-Hill, 1986

11. J.G. Proakis, Digital Communications, Third edition, New York: McGraw-Hill, 1995.

12. L.E. Larson, ed., $R F$ and microwave circuit design for wireless comınunications, Norwood (MA) Artech House, 1996.

13. A.V. Oppenheim, R.W. Schafer, Discrete-Time Signal Processing, Prentice-Hall, 1989.

14. S. Haykin, Adaptive Filter Theory, Prentice-Hall, Englewood Cliffs, N.J., 1992.

15. S.U.H. Qureshi, Adaptive equalization, Proc. IEEE, 73, 1349-1387 (1985).

16. C.A. Belfiore, J.H. Park, Decision feedback equalization, Proc. IEEE, 67, 1143-1156 (1979).

17. G.D. Forney, Maximum-likelihood sequence estimation of digital sequences in the presence of intersymbol interference, IEEE Trans. on Inform. Theory, Vol. IT-18, 363-378 (1972). 
18. G. Ungerboeck, Adaptive maximum-likelihood receiver for carriermodulated data transmission systems, IEEE Trans. on Communications., 22, (May 1974).

19. J.J. Hopfield, Learning algorithms and probability distributions in feedforward and feedback networks, Proc. Nat. Academy Sci., Vol. 84, 8429-8433 (1987).

20. S. Haykin, Neural Networks, a Comprehensive Foundation, Macmillan, New York, NY, 1994.

21. G.E. Hinton, Connectionist learning procedures, Artificial Intelligence, 40, 185-234 (1989).

22. D. Rumelhart, J. McClelland and the PDP Research Group, Parallel Distributed Processing, Vol. 1, The MIT Press, Cambridge, MA, 1986.

23. K.D. Rao, Ch. H. Kumar. M. M. Rao, Improving performance of an adaptive equalizer using EKF trained multilayered neural networks, Journal of IETE, 42, 33-40 (Jan.-Feb. 1996).

24. S. Chen, G.J. Gibson, C.F.N. Cowan, P.M Grant, Reconstruction of binary signals using an adaptive radial-basis-function equalizer, Signal Processing, 22, 77-93 (1991).

25. S. Chen, B. Mülgrew. Overcoming co-channel interference using an adaptive radial basis function equaliser, Signal Processing. 28, 91-107 (1992).

26. T. Kohonen, Self-Organization and associative memory, Series in Information Sciences, Vol. 8. Springer-Verlag, 3rd ed., 1989.

27. T. Kohonen, K. Raivio, O. Simula, O. Ventä, J. Henriksson, Combining linear equalization and self-organizing adaptation in dynamic discretesignal detection, Proc. of the International Joint Conference on Neural Networks, San Diego, CA, Vol. 1, pp. 223-228, Jun. 18-21, 1990.

28. T. Kohonen, K. Raivio, O. Simula, J. Henriksson, Performance evaluation of self-organizing map based neural equalizers in dynamic discrete-signal detection. Proc. of the International Conference on Artificial Neural Networks, Helsinki, Finland, Vol. 2, pp. 1677-1680, Jun. 24-28, 1991.

29. K. Georgoulakis, S. Theodoridis, Efficient clustering techniques for channel equalization in hostile environments, Signal Processing, Vol. $58,153-164$ (1997). 
30. S. Theodoridis, C.F.N. Cowan, C.P. Callender, C. M. S. Lee, Schemes for equalization of communications channels with nonlinear impairments, IEE Proc. Commun. 142, 165-171 (1995).

31. S. Chen, S. McLaughlin, B. Mulgrew, P.M. Grant, Adaptive Bayesian decision feedback equalizer for dispersive mobile radio channels, IEEE Trans. on Communications, 43, 1937-1946 (1995).

32. A. Hussain, J.J. Soraghan, T.S. Durrani, A new adaptive functional-link neural-network-based DFE for overcoming co-channel interference, IEEE Trans. on Communications, 45, 1358-1362 (1997).

33. M. Riedmiller, H. Braun, A direct adaptive method for faster backpropagation learning: the RPROP algorithm, Proc. of IEEE International Conference On Neural Networks, San Francisco, CA, 1993, pp. 586-591.

34. A. K. Jain, R.C. Dubes, Algorithms for Clustering Data. Prentice Hall, 1988.

35. J.T. Tou, R.C. Gonzalez, Pattern Recognition Principles, AddisonWesley, Reading, Massachusetts, 1974.

36. M. Lehtokangas. P. Salmela. J. Saarinen, K. Kaski, Weight initialization techniques, in C. Leondes (Ed.), Algorithms and Architectures, Neural Network Systems Techniques and Applications, Academic Press, 1998, pp. 87-121.

37. A. Kantsila, M. Lehtokangas, J. Saarinen, Improved neural network equalization by the use of maximum covariance weight initialization, Proc. IX European Signal Processing Conference (EUSIPCO'98), Island of Rhodes, Greece, Sep 8-11, 1998, Vol. II, pp. 769-772.

38. E.B. Baum, F. Wilczek, Supervised learning of probability distributions by neural networks, Neural Information Processing Systems, D.Z. Anderson, Ed. New York: Amer. Inst. Physics, 1988. pp. 52-61.

39. N.B. Karayiannis, Accelerating the training of feedforward neural networks using generalized hebbian rules for initializing the internal representations, IEEE Trans. on Neural Networks, 7, 419-426 (1996). 
